\title{
Integral Sliding Mode Controller Design for Stochastic Time-Delay Systems
}

\author{
Michael Basin, Jesus Rodriguez-Gonzalez, Leonid Fridman, Pedro Acosta
}

\begin{abstract}
This paper presents a robustification algorithm for the optimal controller for unobserved linear system states with input delay, linear observations with delay confused with white Gaussian noises, and a quadratic criterion, which is based on integral sliding mode compensation of disturbances. The general principles of the integral sliding mode compensator design are modified to yield the basic control algorithm oriented to time-delay systems, which is then applied to robustify the optimal controller. As a result, the sliding mode compensating control leading to suppression of the disturbances from the initial time moment is designed. The obtained robust control algorithm is verified by simulations in the illustrative example.
\end{abstract}

\section{INTRODUCTION}

This paper presents an integral sliding mode algorithm for robustifying the optimal controller for unobserved linear system states with input delay over linear delayed observations. The integral sliding mode technique was introduced as a method for disturbance suppression from the very beginning of the system functioning [1], [2] and has been successfully applied to robustification of some optimal filters and regulators for time delay systems in [3], [4], [5], where a detailed comment on the up-to-date state of the control and filtering theory for time delay systems has also been given. Comprehensive reviews of theory and algorithms for time delay systems can be found in [6], [7], [8], [9], [10].

The principal result of this paper is the design of an integral sliding mode regulator robustifying the obtained optimal controller for linear systems with input and observation delays and a quadratic criterion, which has recently been obtained in [11], [12], [13]. The idea is to add two compensators, one to the known optimal control and another to the observation process, to suppress external disturbances in the state and observation equations, respectively, that deteriorate the optimal system behavior. The integral sliding mode compensators are realized as relay controls in a such way that the sliding mode motion starts from the initial moment, thus eliminating the external disturbances from the beginning of system functioning [1], [2]. This constitutes the crucial advantage of the integral sliding modes in comparison to the conventional ones. Other original modifications of the sliding mode control technique applicable to disturbance

This work was supported by the Mexican National Science and Technology Council (CONACyT) under Grants 39388-A and 43807-A.

M. Basin and J. Rodriguez-Gonzalez are with Department of Physical and Mathematical Sciences, Autonomous University of Nuevo Leon, Apdo postal 144-F, C.P. 66450, San Nicolas de los Garza, Nuevo Leon, Mexico mbasinafcfm.uanl.mx; jgrg17@hotmail.com.mx

L. Fridman is with National Autonomous University of Mexico Ifridman@verona. fi-p.unam.mx

P. Acosta is with Technological Institute of Chihuahua, Mexico pacosta@platon. itchihuahua.mx suppression were suggested in [14], [15]. Note that in the framework of this modified (in comparison to [4], [5]) integral sliding mode approach, the optimal control is not required to be differentiable and the sliding mode manifold matrix is always invertible. It should be also noted that the compensator in the state equation can simultaneously suppress observation disturbances, as well as the observation compensator can simultaneously suppress state disturbances, if certain matching conditions hold.

The paper is organized as follows. In Section 2, the optimal controller problem is stated and its solution is given for unobserved linear system states with input delay, linear observations with delay confused with white Gaussian noises, and a quadratic criterion. Section 3 outlines the general principles of the integral sliding mode compensator design, which yield the basic control algorithm oriented to time-delay systems. This basic algorithm is then applied to robustify the optimal controller. As a result, two integral sliding mode control compensators are designed in Section 4 to suppress the disturbances in state and observation equations, respectively, from the initial time moment. Section 5 presents an example illustrating the quality of simultaneous disturbance suppression in state and observation equations, provided by the robust integral sliding mode control compensator in the observation equation only, in comparison to performance the optimal controller in the presence of disturbances. Satisfactory results are obtained.

\section{Optimal Controller Problem}

Let $(\Omega, F, P)$ be a complete probability space with an increasing right-continuous family of $\sigma$-algebras $F_{t}, t \geq 0$, and let $\left(W_{1}(t), F_{t}, t \geq t_{0}\right)$ and $\left(W_{2}(t), F_{t}, t \geq t_{0}\right)$ be independent Wiener processes. The partially observed $F_{t}$-measurable random process $(x(t), y(t))$ is described by delay-differential equations for the system state

$$
d x(t)=a(t) x(t) d t+B(t) u(t-\tau) d t+b(t) d W_{1}(t),
$$

with the initial condition $x(s)=\phi(s), s \in\left[t_{0}-\tau, t_{0}\right]$, and the observation process

$$
d y(t)=\left(A_{0}(t)+A(t) x(t-h)\right) d t+F(t) d W_{2}(t) .
$$

Here, $x(t) \in R^{n}$ is the state vector, $u(t) \in R^{m}$ is the control input, $y(t) \in R^{p}$ is the observation process, $\phi(s)$ is a mean square piecewise-continuous Gaussian stochastic process (see [16] for definition) given in the interval $\left[t_{0}-\tau, t_{0}\right]$ such that $\phi(s), W_{1}(t)$, and $W_{2}(t)$ are independent.

The observation process $y(t)$ depends on the delayed state $x(t-h)$, where $h$ is an observation delay, which assumes that collection of information on the system state for the 
observation purposes is possible only after a certain time $h$. It is assumed that $A(t)$ is a nonzero matrix and $F(t) F^{T}(t)$ is a positive definite matrix. All coefficients in (1)-(2) are deterministic functions of appropriate dimensions. The quadratic cost function $J$ to be minimized is defined as follows

$$
\begin{gathered}
J=E\left[\frac{1}{2} x^{T}(T) \Phi x(T)+\right. \\
\left.\frac{1}{2} \int_{t_{0}}^{T} u^{T}(s) R(s) u(s) d s+\frac{1}{2} \int_{t_{0}}^{T} x^{T}(s) L(s) x(s) d s\right]
\end{gathered}
$$

where $K$ is positive definite and $\Phi, L$ are nonnegative definite symmetric matrices, $T>t_{0}$ is a certain time moment, the symbol $E[f(x)]$ means the expectation (mean) of a function $f$ of a random variable $x$, and $a^{T}$ denotes transpose to a vector (matrix) $a$.

The optimal controller problem is to find the control $u^{*}(t), t \in\left[t_{0}, T\right]$, that minimizes the criterion $J$ along with the unobserved trajectory $x^{*}(t), t \in\left[t_{0}, T\right]$, generated upon substituting $u^{*}(t)$ into the state equation (1).

The following solution to this problem was obtained (see [11], [12], [13]). The optimal control law is given by

$$
u^{*}(t-\tau)=(R(t))^{-1} B^{T}(t) \exp \left(-\int_{t-\tau}^{t} a^{T}(s) d s\right) Q(t) m(t-\tau),
$$

where matrix function $Q(t)$ is the solution of the Riccati equation

$$
\begin{gathered}
\dot{Q}(t)=-a^{T}(t) Q(t)-Q(t) a(t)+L(t)-Q(t) \times \\
\exp \left(-\int_{t-\tau}^{t} a(s) d s\right) B(t) R^{-1}(t) B^{T}(t) \exp \left(-\int_{t-\tau}^{t} a^{T}(s) d s\right) Q(t),
\end{gathered}
$$

with the terminal condition $Q(T)=\Phi$. Upon substituting the optimal control (4) into the optimal estimate equation, the following optimally controlled state estimate equation is obtained

$$
\begin{gathered}
d m(t)=\left(a(t) m(t)+B(t)\left(R(t)^{-1} B^{T}(t) \exp \left(-\int_{t-\tau}^{t} a^{T}(s) d s\right) \times\right.\right. \\
Q(t) m(t-\tau)) d t+P(t) \exp \left(-\int_{t-h}^{t} a^{T}(s) d s\right) \times \\
A^{T}(t)\left(F(t) F^{T}(t)\right)^{-1}\left(d y(t)-\left(A_{0}(t)+A(t) m(t-h)\right) d t\right),
\end{gathered}
$$

with the initial condition $m(s)=E(\phi(s)), s \in\left[t_{0}-\tau, t_{0}\right)$ and $m\left(t_{0}\right)=E\left(\phi\left(t_{0}\right) \mid F_{t_{0}}^{Y}\right), s=t_{0}$, where the error variance matrix $P(t)$ satisfies the Riccati equation

$$
\begin{gathered}
d P(t)=\left(P(t) a^{T}(t)+a(t) P(t)+b(t) b^{T}(t)-\right. \\
P(t) \exp \left(-\int_{t-h}^{t} a^{T}(s) d s\right) A^{T}(t)\left(F(t) F^{T}(t)\right)^{-1} \times \\
\left.A(t) \exp \left(-\int_{t-h}^{t} a(s) d s\right) P(t)\right) d t .
\end{gathered}
$$

with the initial condition $P\left(t_{0}\right)=E\left(\left(x\left(t_{0}\right)-m\left(t_{0}\right)\right)\left(x\left(t_{0}\right)-\right.\right.$ $\left.\left.m\left(t_{0}\right)\right)^{T} \mid y\left(t_{0}\right)\right)$.

Thus, the optimally controlled state estimate equation (6), the gain matrix constituent equation (5), the optimal control law (4), and the variance equation (7) give the complete solution to the optimal controller problem for unobserved states of linear systems with control and observation delays.

\section{ROBUST CONTROL PROBLEM}

In practical applications, a control system operates under uncertainty conditions that may be generated by parameter variations or external disturbances. Consider a real trajectory of the disturbed control system

$$
\dot{x}(t)=f(x(t))+B(t) u+g_{1}(x(t), t)+g_{2}(x(t-\tau), t) .
$$

Here $u \in R^{m}$ is the control input, the rank of matrix $B(t)$ is complete and equal to $m$ for any $t>t_{0}$, and the pseudoinverse matrix of $B$ is uniformly bounded:

$\left\|B^{+}(t)\right\| \leq b^{+}, b^{+}=$const $>0, B^{+}(t):=\left[B^{T}(t) B(t)\right]^{-1} B^{T}(t)$, and $B^{+}(t) B(t)=I$, where $I$ is the $m$-dimensional identity matrix. Uncertain inputs $g_{1}$ and $g_{2}$ represent smooth disturbances corresponding to perturbations and nonlinearities in the system. For $g_{1}, g_{2}$, the standard matching conditions are assumed to be held: $g_{1}, g_{2} \in \operatorname{span} B$, or, in other words, there exist smooth functions $\gamma_{1}, \gamma_{2}$ such that

$$
\begin{gathered}
g_{1}(x(t), t)=B(t) \gamma_{1}(x(t), t), \\
g_{2}(x(t-\tau), t)=B(t) \gamma_{2}(x(t-\tau), t), \\
\left\|\gamma_{1}(x(t), t)\right\| \leq q_{1}\|x(t)\|+p_{1}, q_{1}, p_{1}>0, \\
\left\|\gamma_{2}(x(t-\tau), t)\right\| \leq q_{2}\|x(t-\tau)\|+p_{2}, q_{2}, p_{2}>0 .
\end{gathered}
$$

The last two conditions provide reasonable restrictions on the growth of the uncertainties.

Let us also consider the nominal control system

$$
\dot{x}_{0}(t)=f\left(x_{0}(t)\right)+B(t) u_{0}\left(x_{0}(t-\tau), t\right),
$$

where a certain delay-dependent control law $u_{0}(x(t-\tau), t)$ is realized. The problem is to reproduce the nominal state motion determined by (10) in the disturbed control system (8).

The following initial conditions are assumed for the system (10)

$$
x(s)=\phi(s),
$$

where $\phi(s)$ is a piecewise continuous function given in the interval $\left[t_{0}-\tau, t_{0}\right]$.

Thus, the control problem now consists in robustification of control design in the nominal system (10) with respect to uncertainties $g_{1}, g_{2}$ : to find such a control law $u=u_{0}(x(t-$ $\tau), t)+u_{1}(t)$ that the disturbed trajectories (8) with initial conditions (11) coincide with the nominal trajectories (10) with the same initial conditions (11).

\section{A. Design principles for state disturbance compensator}

Let us design the control law for (8) in the form

$$
u(t)=u_{0}(x(t-\tau), t)+u_{1}(t),
$$

where $u_{0}(x(t-\tau), t)$ is the nominal feedback control designed for (10), and $u_{1}(t) \in R^{m}$ is the relay control generating the integral sliding mode in some auxiliary space to reject uncertainties $g_{1}, g_{2}$. Substitution of the control law (12) into the system (8) yields

$$
\dot{x}(t)=f(x(t))+B(t) u_{0}(x(t-\tau), t)+B(t) u_{1}(t)+
$$




$$
g_{1}(x(t), t)+g_{2}(x(t-\tau), t) .
$$

Define the auxiliary function

$$
s(t)=z(t)+s_{0}(x(t), t),
$$

where $s_{0}(x(t), t)=B^{+}(t) x(t)$, and $z(t)$ is an auxiliary variable defined below. Then,

$$
\dot{s}(t)=\dot{z}(t)+G(t)\left[f(x(t))+B(t) u_{0}(x(t-\tau), t)+\right.
$$

$\left.\left.B\left(\gamma_{1}(x(t), t)\right)+\gamma_{2}(x(t-\tau), t)\right)+B(t) u_{1}(t)\right]+\left(\partial s_{0}(x(t), t) / \partial t\right)$, $G(t)=\partial s_{0}(x(t), t) / \partial x=B^{+}(t) \quad$ and $\quad \partial s_{0}(x(t), t) / \partial t=$ $\left(d\left(B^{+}(t)\right) / d t\right) x(t)$. Note that in the framework of this modified (with respect to [4], [5]) integral sliding mode approach, the optimal control $u_{0}(x(t))$ is not required to be differentiable and the sliding mode manifold matrix $G B=B^{+} B=I$ is always invertible.

The philosophy of integral sliding mode control is the following: in order to achieve $x(t)=x_{0}(t)$ at all $t \in\left[t_{0}, \infty\right)$, the sliding mode should be organized on the surface $s(t)$, since the following disturbance compensation should have been obtained in the sliding mode motion

$$
\begin{gathered}
B^{+}(t) B(t) u_{1 e q}(t)= \\
-B^{+}(t) B(t) \gamma_{1}(x(t), t)-B^{+}(t) B(t) \gamma_{2}(x(t-h), t),
\end{gathered}
$$

that is

$$
u_{1 e q}(t)=-\gamma_{1}(x(t), t)-\gamma_{2}(x(t-h), t) .
$$

Note that the equivalent control $u_{1 e q}(t)$ can be unambiguously determined from the last equality and the initial condition for $x(t)$.

Define the auxiliary variable $z(t)$ as the solution to the differential equation

$$
\begin{gathered}
\dot{z}(t)=-B^{+}(t)\left[f(x(t))+B(t) u_{0}(x(t-\tau), t)\right]+ \\
d\left(\left(B^{+}(t)\right) / d t\right) x(t),
\end{gathered}
$$

with the initial condition $z(0)=-s_{0}(0)=-B^{+}(0) \varphi(0)$. Then, the sliding manifold equation takes the form

$$
\begin{gathered}
\left.\dot{s}(t)=B^{+}(t)\left[B(t)\left(\gamma_{1}(x(t), t)\right)+\gamma_{2}(x(t-\tau), t)\right)+B(t) u_{1}(t)\right]= \\
=\gamma_{1}(x(t), t)+\gamma_{2}(x(t-\tau), t)+u_{1}(t)=0 .
\end{gathered}
$$

Finally, to realize sliding mode, the relay control is designed

$$
\begin{gathered}
u_{1}(t)=-M(x(t), x(t-\tau), t) \operatorname{sign}[s(t)], \\
M=q(\|x(t)\|+\|x(t-\tau)\|)+p,
\end{gathered}
$$

$q>q_{1}, q_{2}, p>p_{1}+p_{2}$.

The convergence to and along the sliding mode manifold $s(t)=0$ is assured by the Lyapunov function $V(t)=$ $s^{T}(t) s(t) / 2$ for the system (9) with the control input $u_{1}(t)$ of (16):

$$
\begin{gathered}
\dot{V}(t)=s^{T}(t)\left[\gamma_{1}(x(t), t)+\gamma_{2}(x(t-h), t)+u_{1}(t)\right] \leq \\
-|s(t)|([q(\|x(t)\|+\|x(t-\tau)\|)+p]+ \\
\left.\left[\gamma_{1}(x(t), t)+\gamma_{2}(x(t-\tau), t)\right]\right)<0,
\end{gathered}
$$

where $|s(t)|=\sum_{i=1}^{m}\left|s_{i}(t)\right|$.
B. Design principles for observation disturbance compensator

Let the observation process (2) be corrupted with unknown disturbances

$$
\begin{gathered}
d y(t)=\left(A_{0}(t)+A(t) x(t-h)\right) d t+F(t) d W_{2}(t)+ \\
\left(k_{1}(x(t), t)+k_{2}(x(t-h), t)\right) d t,
\end{gathered}
$$

where $k_{1}(x(t), t)$ and $k_{2}(x(t-h), t)$ are non-Gaussian and, possibly, deterministic noises not bearing any useful information and depending on the current and delayed states. Such disturbances obviously deteriorate the quality of estimation and should be eliminated.

For this purpose, assume that the disturbances satisfy the following conditions (note that no matching conditions are assumed)

$$
\begin{gathered}
\left\|k_{1}(x(t), t)\right\| \leq q_{3}\|x(t)\|+p_{3}, q_{3}, p_{3}>0, \\
\left\|k_{2}(x(t-h), t)\right\| \leq q_{4}\|x(t-h)\|+p_{4}, q_{4}, p_{4}>0,
\end{gathered}
$$

providing reasonable restrictions on their growth.

The observation process (17) consists of the useful and parasitic parts, $y(t)=y_{u}(t)+y_{p}(t)$, where $d y_{u}(t)=\left(A_{0}(t)+\right.$ $A(t) x(t-h)) d t+B(t) d W_{2}(t)$ and $d y_{p}(t)=\left(g_{1}(x(t), t)+\right.$ $\left.g_{2}(x(t-h), t)\right) d t$. If only the useful signal $y_{u}(t)$ is present, the optimal filter based on the observations $y_{u}(t)$ yields the desirable estimate $m_{u}(t)$ for the unobserved state $x(t)$. At this point, the problem is to suggest a tuning adjustment $y_{1}(t)$ that, being added to the actual observations $y(t)=$ $y_{u}(t)+y_{p}(t)$, compensates for observation disturbances $k_{1}$, $k_{2}$.

The following sliding mode technique solves this problem: define the sliding manifold $s(t)$ as $s(t)=z(t)+s_{0}(t)$, where $s_{0}(t)=m(t)$ and $z(t)$ is an auxiliary variable to be assigned. The condition of motion along the sliding manifold, $d s(t) / d t=0$, yields:

$$
\begin{gathered}
d s(t)=d z(t)+\left(E\left(f(x(t)) \mid F_{t_{0}}^{Y}\right)+B(t) u(t-\tau)\right) d t+ \\
C(t)\left[d y(t)-\left(A_{0}(t)+A(t) m(t-h)\right) d t+y_{1}(t) d t\right]=0,
\end{gathered}
$$

where $y(t)$ is the disturbed observation process (17), $C(t)=$ $P(t) \exp \left(-\int_{t-h}^{t} a^{T}(s) d s\right) A^{T}(t) \quad\left(F(t) F^{T}(t)\right)^{-1}$ is the filter gain matrix, and $y_{1}(t)$ is the tuning adjustment to observations, whose values on the sliding manifold are denoted by $y_{\text {leq }}(t)$. The value of the tuning adjustment on the sliding manifold must be equal to

$$
y_{1 e q}(t)=-\left(k_{1}(x(t), t)+k_{2}(x(t-h), t)\right),
$$

thus compensating for unknown disturbances. In doing so, in view of $d s(t) / d t=d z(t) / d t+d s_{0}(t) / d t$, the auxiliary variable $z(t)$ is assigned by the equation

$$
\begin{gathered}
d z(t)=\left(-E\left(f(x(t)) \mid F_{t_{0}}^{Y}\right)-B(t) u(t-\tau)\right) d t- \\
C(t)\left[d y_{u}(t)-\left(A_{0}(t)+A(t) m(t-h)\right) d t\right],
\end{gathered}
$$

with the initial condition $z(0)=-s_{0}(0)=-m(0)$, where $y_{u}(t)$ is an average of some a priori known realizations of the useful signal. Thus, the estimate $m(t)$ based on the 
disturbed observations with compensator $y(t)+y_{1}(t)$ and the desired estimate $m_{u}(t)$ coincide in the mean square as random variables on the sliding manifold $s(t)=0$, and the sliding manifold equation takes the form

$$
\dot{s}(t)=k_{1}(x(t), t)+k_{2}(x(t-h), t)+y_{1 e q}(t)=0,
$$

thus assuring compensation of the observation disturbances.

Finally, to realize sliding mode, the relay compensator control is designed

$$
\left.y_{1}(t)=-M(x(t), x(t-h), t)\right) \operatorname{sign}[s(t)],
$$

where $M=\tilde{q}(\|x(t)\|+\|x(t-h)\|)+\tilde{p}, \tilde{q}>q_{3}, q_{4}, \tilde{p}>p_{3}+$ $p_{4}$. The mean square convergence to and along the sliding mode manifold is proved using the same Lyapunov function as in the preceding subsection.

\section{ROBUST SLIDING MODE CONTROLLER DESIGN FOR LINEAR SYSTEM WITH INPUT AND OBSERVATION DELAYS}

Consider the disturbed state equation (1), whose behavior is affected by uncertainties $g_{1}, g_{2}$, presenting perturbations and nonlinearities in the system

$$
\begin{gathered}
d x(t)=a(t) x(t) d t+B(t) u(t-\tau) d t+ \\
b(t) d W_{1}(t)+g_{1}(x(t), t)+g_{2}(x(t-h), t),
\end{gathered}
$$

with the initial condition $x(s)=\phi(s), s \in\left[t_{0}-\tau, t_{0}\right]$, and the observation equation (2), which is now affected by uncertainties $k_{1}, k_{2}$, presenting perturbations and nonlinearities in the observations (2)

$$
\begin{gathered}
d y(t)=\left(A_{0}(t)+A(t) x(t-h)\right) d t+F(t) d W_{2}(t)+ \\
\left(k_{1}(x(t), t)+k_{2}(x(t-h), t)\right) d t .
\end{gathered}
$$

It is also assumed that the uncertainties satisfy the standard matching and growth conditions (9) given in Section 3, and the quadratic cost function (2) is the same as in Section 2.

The optimally controlled estimate equation (7) for the state (21) over the observations (22) takes the form

$$
\begin{gathered}
d m(t)=\left(a(t) m(t)+B(t)\left(R(t)^{-1} B^{T}(t) \times\right.\right. \\
\exp \left(-\int_{t-\tau}^{t} a^{T}(s) d s\right) Q(t) m(t-\tau)+B g_{1}(x(t), t)+ \\
\left.B g_{2}(x(t-h), t)\right) d t+P(t) \exp \left(-\int_{t-h}^{t} a^{T}(s) d s\right) A^{T}(t) \times \\
\left(F(t) F^{T}(t)\right)^{-1}\left(d y(t)-\left(A_{0}(t)+A(t) m(t-h)\right) d t\right),
\end{gathered}
$$

with the initial condition $m(s)=E(\phi(s)), s \in\left[t_{0}-\tau, t_{0}\right)$ and $m\left(t_{0}\right)=E\left(\phi\left(t_{0}\right) \mid F_{t_{0}}^{Y}\right), s=t_{0}$.

The problem is to robustify the obtained optimal controller (4)-(7), using the methods specified by (14)-(16) and (18)(20). First, define the new control (12) as follows: $u(t)=$ $u_{0}(x(t-\tau), t)+u_{1}(t)$, where the optimal control $u_{0}(x(t-$ $\tau), t$ ) coincides with (4) and the robustifying component $u_{1}(t)$ is obtained according to (16)

$$
\begin{gathered}
u_{1}(t)=-M(x(t), x(t-\tau), t) \operatorname{sign}[s(t)], \\
M=q(\|x(t)\|+\|x(t-\tau)\|)+p,
\end{gathered}
$$

$q>q_{1}, q_{2}, p>p_{1}+p_{2}$. Consequently, the sliding mode manifold function $s(t)$ is defined as $s(t)=z(t)+s_{0}(x(t), t)$, where $s_{0}(m(t), t)=B^{+}(t) m(t)$, and the auxiliary variable $z(t)$ satisfies the delay differential equation

$$
\begin{gathered}
d z(t)=-B^{+}(t)[a(t) m(t) d t+ \\
B(t)\left(R(t)^{-1} B^{T}(t) \exp \left(-\int_{t-\tau}^{t} a^{T}(s) d s\right) Q(t) m(t-\tau) d t+\right. \\
\left.C(t)\left(d y(t)-\left(A_{0}(t)+A(t) m(t-h)\right) d t\right)\right]
\end{gathered}
$$

with the initial condition $\left.z(0)=-B^{+}(0) \varphi(0)\right)$, where $C(t)=$ $P(t) \exp \left(-\int_{t-h}^{t} a^{T}(s) d s\right) A^{T}(t)\left(F(t) F^{T}(t)\right)^{-1}$.

Note that the compensator $u_{1}(t)$ introduced at this step can also compensate for observation disturbances $k_{1}, k_{2}$, if the filter gain matrix $C(t)$ belongs to the matrix $B(t)$ span, i.e., $C(t)=B(t) M(t)$. This fact readily follows from the sliding mode equation (15) and the structure of the disturbed controlled estimate equation (23).

Thus, the introduced control $u_{1}(t)$ can compensate for state disturbances $g_{1}, g_{2}$ and observation disturbances $k_{1}, k_{2}$, if the matching condition $C(t)=B(t) M(t)$ holds. However, the compensator (20) should still be applied to compensate for observation disturbances in the unmatched case. For this purpose, define the new observation process $y(t)+y_{1}(t)$, where $y(t)$ are actual observations, and the robustifying component $y_{1}(t)$ is obtained according to (20)

$$
\left.y_{1}(t)=-M(x(t), x(t-h), t)\right) \operatorname{sign}[s(t)],
$$

where $M=\tilde{q}(\|x(t)\|+\|x(t-h)\|)+\tilde{p}, \tilde{q}>q_{3}, q_{4}, \tilde{p}>p_{3}+$ $p_{4}$. Consequently, the sliding mode manifold function $s(t)$ is defined as $s(t)=z(t)+s_{0}(t)$, where $s_{0}(t)=m(t)$, and the auxiliary variable $z(t)$ satisfies the delay differential equation

$$
\begin{gathered}
d z(t)=(-a(t) m(t)- \\
B(t)\left(R(t)^{-1} B^{T}(t) \exp \left(-\int_{t-\tau}^{t} a^{T}(s) d s\right) Q(t) m(t-\tau)\right) d t- \\
C(t)\left[d y_{u}(t)-\left(A_{0}(t)+A(t) m(t-h)\right) d t\right]
\end{gathered}
$$

with the initial condition $z(0)=-s_{0}(0)=-m(0)$. The undisturbed observations $y_{u}(t)$ could be determined from the nominal system corresponding to (21),(22), where all disturbances and white noises are absent and the initial condition for (21) coincides with $m_{0}$.

Note that the compensator $y_{1}(t)$ introduced at this step can also compensate for state disturbances $g_{1}, g_{2}$, if the state disturbances satisfy the matching conditions with the filter gain matrix $C(t)$, i.e.,

$$
\begin{gathered}
g_{1}(x(t), t)=C(t) \beta_{1}(x(t), t), \\
g_{2}(x(t-h), t)=C(t) \beta_{2}(x(t-h), t) .
\end{gathered}
$$

This fact readily follows from the sliding mode equation (18) and the structure of the disturbed controlled estimate equation (23). A case of joint compensation of state and observation disturbances using the only observation disturbance compensator (20) is presented in the next section. 


\section{EXAMPLE}

Let us consider a scalar linear system

$$
\dot{x}(t)=x(t)+u(t-0.1), \quad x(s)=\phi(s), s \in[-0.1,0],
$$

where $\phi(s)=N(0,1)$ for $s \in[-0.1,0]$, and $N(0,1)$ is a Gaussian random variable with zero mean and unit variance. The observation process is given by

$$
y(t)=x(t-0.2)+\psi(t),
$$

where $\psi(t)$ is a white Gaussian noise, which is the weak mean square derivative of a standard Wiener process (see [16]). The equations (24) and (25) present the conventional form for the equations (1) and (2), which is actually used in practice [17].

The control problem is to find the control $u(t), t \in[0, T]$, that minimizes the criterion

$$
J=E\left\{\frac{1}{2}\left[x(T)-x^{*}\right]^{2}+\frac{1}{2} \int_{0}^{T} u^{2}(t) d t\right\},
$$

where $T=0.45$, and $x^{*}=25$ is a large value of $x(t)$, which would a priori be unreachable for the optimally controlled system at the time $T$. In other words, the control problem is to maximize the unobserved state $x(t)$ using the minimum energy of control $u$.

Let us apply the optimal controller (4)-(7) for linear systems with control and observation delays to the system (24),(25). Since $\exp \left(-\int_{t-h}^{t} a^{T}(s) d s\right)=\exp (-0.2)$ and $\exp \left(-\int_{t-\tau}^{t} a^{T}(s) d s\right)=\exp (-0.1)$, the control law (4) takes the form

$$
u^{*}(t-0.1)=(\exp (-0.1)) Q(t) m(t-0.1)
$$

where

$$
\begin{gathered}
\dot{m}(t)=m(t)+u(t-0.1)+ \\
(\exp (-0.2)) P(t)(y(t)-m(t-0.2)), \\
m(s)=0, s<0, m(0)=m_{0}, \\
\dot{Q}(t)=-2 Q(t)-(\exp (-0.1) Q(t))^{2}, \quad Q(0.45)=1, \\
\dot{P}(t)=2 P(t)-(\exp (-0.2) P(t))^{2}, \quad P(0)=P_{0} .
\end{gathered}
$$

Upon substituting the control (27) into (28), the optimally controlled estimate equation takes the form

$$
\begin{gathered}
\dot{m}(t)=m(t)+(\exp (-0.1)) Q(t) m(t-0.1)+ \\
(\exp (-0.2)) P(t)(y(t)-m(t-0.2)), \\
m(s)=0, s<0, m(0)=m_{0} .
\end{gathered}
$$

For numerical simulation of the system (24),(25), the initial value $x(0)=0.05$ is assigned for realization of the Gaussian variable $x(0)=\phi(0)$ in (24), the values $m_{0}=1.9$ and $P_{0}=10$ are assigned as the initial conditions of the estimate $m(t)$ and the filter gain $P(t)$, respectively, and the disturbance $\psi(t)$ in (25) is realized using the built-in MatLab white noise function.

The results of applying the controller (4)-(7) to the system (24),(25) are shown in Fig. 1, which presents the graphs of the state (24) $x(t)$ controlled by (27), the controlled estimate
(31) $m(t)$, the criterion (26) $J(t)$, the control (27) $u^{*}(t)$, the variance (30) $P(t)$, and the control gain (29) $Q(t)$, in the interval $[0, T]$. The values of the state $(24)$, the controlled estimate (28), and the criterion (26) at the final moment $T=$ 0.45 are $x(0.45)=6.87, m(0.45)=6.876$, and $J(0.45)=$ 215.43.

The next task is to introduce state and observation disturbances into the controlled system (24). These disturbances are realized as a constant: $g(t)=k(t)=100$. The matching conditions are valid, because state $x(t)$, control $u(t)$, and observations $y(t)$ have the same dimension: $\operatorname{dim}(x)=$ $\operatorname{dim}(u)=\operatorname{dim}(y)=1$. The restrictions on the disturbance growth hold with $q_{1}=q_{2}=p_{2}=q_{3}=q_{4}=p_{4}=0$ and $p_{1}=p_{3}=100$, since $\|g(t)\|=\|k(t)\|=100$. The disturbed controller equation (31) takes the form

$$
\begin{gathered}
\dot{m}(t)=m(t)+(\exp (-0.1)) Q(t) m(t-0.1)+100+ \\
(\exp (-0.2)) P(t)(y(t)-m(t-0.2)+100), \\
m(s)=0, s<0, m(0)=m_{0} .
\end{gathered}
$$

The system behavior significantly deteriorates upon introducing the disturbances. Figure 2 presents the graphs of the state (24) $x(t)$ controlled by (27), the controlled estimate (32) $m(t)$, the criterion (26) $J(t)$, and the control (27) $u(t)$, in the interval $[0, T]$. The value of the state (24), the controlled estimate (32) and the criterion (26) at the final moment $T=0.45$ are $x(0.45)=255.7, m(0.45)=511$, and $J(0.45)=96660$. The deterioration of the criterion value in comparison to that obtained using the optimal controller (28) is more than 300 times.

Let us finally design the robust integral sliding mode observation disturbance compensator for the introduced disturbances. The new controlled state equation should be

$$
\begin{gathered}
\dot{m}(t)=m(t)+(\exp (-0.1)) Q(t) m(t-0.1)+100+ \\
(\exp (-0.2)) P(t)\left(y(t)-m(t-0.2)+100+y_{1}(t)\right), \\
m(s)=0, s<0, m(0)=m_{0} .
\end{gathered}
$$

where the compensator $y_{1}(t)$ is obtained according to (24)

$$
y_{1}(t)=-M(x(t), x(t-h), t) \operatorname{sign}[s(t)],
$$

and $M=230.4>p_{1}(\exp (0.2))\left(\max _{t \leq 0.45}\left(P^{-1}(t)\right)\right)+p_{3}$. The sliding mode manifold $s(t)$ is defined by $s(t)=z(t)+s_{0}(t)$, where $s_{0}(t)=m(t)$.

The auxiliary variable $z(t)$ satisfies the delay differential equation

$$
\begin{gathered}
\dot{z}(t)=-m(t)-(\exp (-0.1)) Q(t) m(t-0.1)- \\
(\exp (-0.2)) P(t)\left(y_{u}(t)-m(t-0.2)\right),
\end{gathered}
$$

with the initial condition $z(0)=-m(0)=-1.9$, where the undisturbed observations $y_{u}(t)$ are determined from the undisturbed system (24),(25)

$$
\begin{gathered}
\dot{x}_{u}(t)=x(t)+(\exp (-0.1)) Q(t) x_{u}(t-0.1), \\
y_{u}(t)=x_{u}(t-0.2),
\end{gathered}
$$


with the initial condition $x_{u}(s)=0, s<0, x_{u}(0)=m_{0}$.

Upon introducing the compensator (34) into the controller equation (33), the controlled estimate behavior is very much improved. Figure 3 presents the graphs of of the state (24) $x(t)$ controlled by (27), the controlled estimate (33) $m(t)$, the criterion (26) $J(t)$, and the control (27) $u(t)$, after applying the compensator (34), in the interval $[0, T]$. The value of the state (24), the controlled estimate (33) and the criterion (26) at the final moment $T=0.45$ are $x(0.45)=8.087$, $m(0.45)=8.066$, and $J(0.45)=215.31$. Thus, the values of the criterion and state after applying the compensator (43) are even better than those for the controller (31), although approximation of the true state by the estimate $m(t)$ is a bit worse. This phenomenon is produced by difference in random realizations of the observation white noise in (25) in both cases.

\section{REFERENCES}

[1] V.I. Utkin, J. Guldner, and J. Shi, Sliding Mode Control in Electromechanical Systems, Taylor and Francis, London (1999).

[2] J.-X. Xu, Y.-J. Pan, and T.-H. Lee, Analysis and design of integral sliding mode control based on Lyapunov's direct method, in Proc. American Control Conference 2003 (Denver, CO, June 2003), pp. 192196 (2003).

[3] M. V. Basin, L. M. Fridman, and M. Skliar, Optimal and robust sliding mode filter for systems with continuous and delayed measurements, in Proc. 41st Conference on Decision and Control (Las Vegas, NV, December 10-13, 2002), pp. 2594-2599 (2002).

[4] M. V. Basin, L. M. Fridman, P. Acosta, and J. Rodriguez-Gonzalez, Robust integral sliding mode regulator for linear systems with time delay in control input, in Proc. American Control Conference 2003 (Denver, CO, June 4-6, 2003), pp. 2138-2143 (2003).

[5] M. V. Basin, L. M. Fridman, J.G. Rodriguez-Gonzalez, and P. Acosta, Optimal and robust sliding mode control for linear systems with multiple time delays in control input, Asian Journal of Control, Vol. 5, no. 4, pp. 557-567 (2003).

[6] M. Malek-Zavarei and M. Jamshidi, Time-Delay Systems: Analysis, Optimization and Applications, North-Holland, Amsterdam, 1987.

[7] V. B. Kolmanovskii and L. E. Shaikhet, Control of Systems with Aftereffect, American Mathematical Society, Providence, 1996.

[8] V. B. Kolmanovskii and A. D. Myshkis, Introduction to the Theory and Applications of Functional Differential Equations, Kluwer, New York, 1999.

[9] M. S. Mahmoud, Robust Control and Filtering for Time-Delay Systems, Marcel Dekker, 2000.

[10] E.-K. Boukas and Z.-K. Liu, Deterministic and Stochastic Time-Delay Systems, Birkhauser, 2002.

[11] M. V. Basin and R. Martinez-Zuniga, Optimal linear filtering over observations with multiple delays, International Journal of Robust and Nonlinear Control, Vol. 14, no. 8, pp. 685-696 (2004).

[12] M. V. Basin, J. Rodriguez-Gonzalez, and R. Martinez-Zuniga, Optimal control for linear systems with time delay in control input, Journal of The Franklin Institute, Vol. 341, no. 3, pp. 267-278 (2004). Special Issue on the 2003 Franklin Institute Awards.

[13] M. V. Basin, J. Rodriguez-Gonzalez, and R. Martinez-Zuniga, Optimal controller for linear systems with input and observation delays, Dynamics of Continuous, Discrete, and Impulsive Systems, Vol. 12B, no. 1, pp. 1-11 (2005).

[14] Y. B. Shtessel, A. S. I. Zinober, and I. Shkolnikov, Sliding mode control for nonlinear systems with output delay via method of stable system center, ASME Trans. J. Dyn. Syst. Measur. Contr., Vol. 125, no. 2, pp. 253-257 (2003).

[15] Y. Orlov, W. Perruquetti, and J.-P. Richard, Sliding mode control synthesis of uncertain time-delay systems, Asian Journal of Control, Vol. 5, no. 4, pp. 568-577 (2003).

[16] V. S. Pugachev and I. N. Sinitsyn, Stochastic Systems: Theory and Applications, World Scientific, 2001.

[17] K.J. Åström, Introduction to Stochastic Control Theory, Academic Press, New York, 1970.
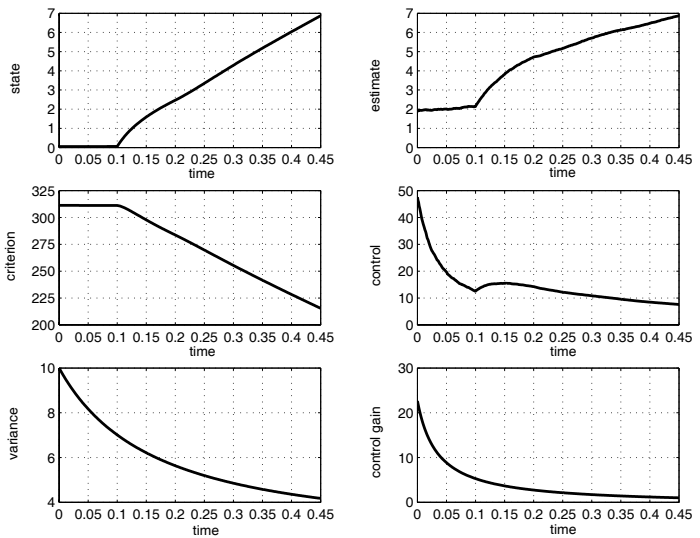

Fig. 1. Graphs of the state (24) $x(t)$ controlled by the optimal linear regulator (27) designed for systems with input and observation delays, the controlled estimate (31) $m(t)$, the criterion (26) $J(t)$, the control (27) $u^{*}(t)$, the variance (30) $P(t)$, and the control gain (29) $Q(t)$, in the interval $[0,0.45]$.
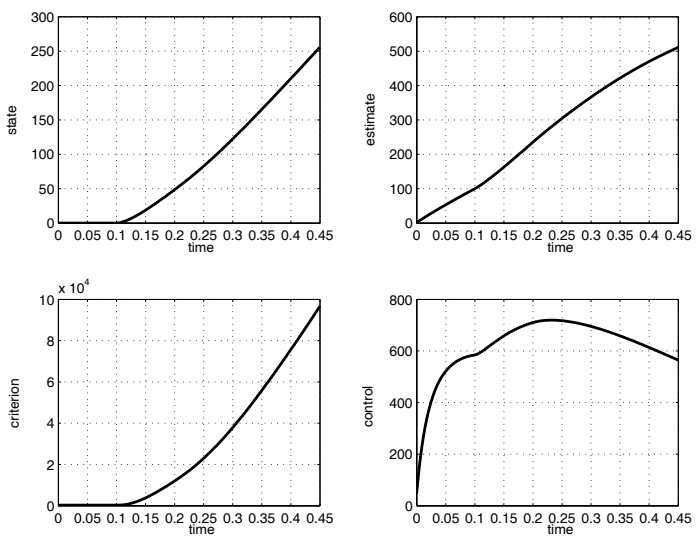

Fig. 2. Controlled system in the presence of disturbance. Graphs of the disturbed state (24) $x(t)$, the disturbed controlled estimate (32) $m(t)$, the disturbed criterion (26) $J(t)$, and the disturbed control (27) $u(t)$ in the interval $[0,0.45]$.
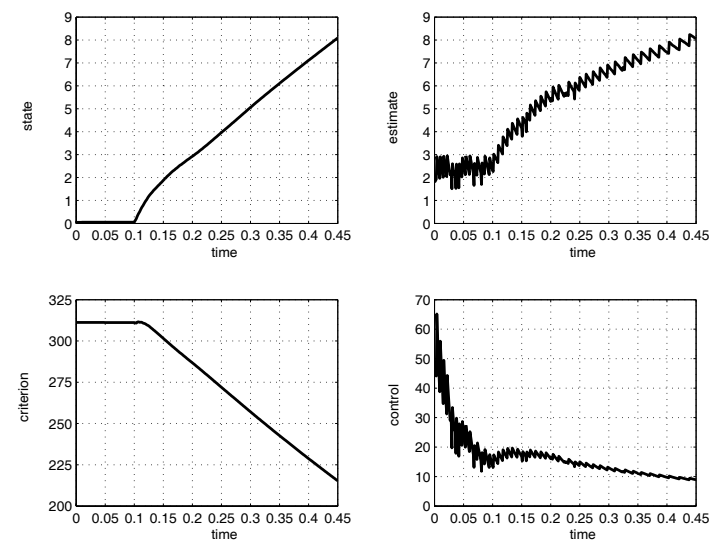

Fig. 3. Controlled system after applying robust integral sliding mode compensator. Graphs of the compensated state (24) $x(t)$, the compensated controlled estimate (33) $m(t)$, the compensated criterion (26) $J(t)$, and the compensated control (27) $u(t)$ in the interval $[0,0.45]$. 